\title{
Increased skeletal muscle mitochondrial efficiency in rats with fructose-induced alteration in glucose tolerance
}

\author{
Raffaella Crescenzo, Francesca Bianco, Paola Coppola, Arianna Mazzoli, Luisa Cigliano, \\ Giovanna Liverini and Susanna Iossa* \\ Department of Biology, Complesso Universitario di Monte Sant'Angelo, Edificio 7, Via Cinthia, I-80126 Napoli, Italy
}

(Submitted 7 November 2012 - Final revision received 18 April 2013 - Accepted 22 April 2013 - First published online 22 May 2013)

\begin{abstract}
In the present study, the effect of long-term fructose feeding on skeletal muscle mitochondrial energetics was investigated. Measurements in isolated tissue were coupled with the determination of whole-body energy expenditure and insulin sensitivity. A significant increase in plasma NEFA, as well as in skeletal muscle TAG and ceramide, was found in fructose-fed rats compared with the controls, together with a significantly higher plasma insulin response to a glucose load, while no significant variation in plasma glucose levels was found. Significantly lower RMR values were found in fructose-fed rats starting from week 4 of the dietary treatment. Skeletal muscle mitochondrial mass and degree of coupling were found to be significantly higher in fructose-fed rats compared with the controls. Significantly higher lipid peroxidation was found in fructose-fed rats, together with a significant decrease in superoxide dismutase activity. Phosphorylated Akt levels normalised to plasma insulin levels were significantly lower in fructose-fed rats compared with the controls. In conclusion, a fructose-rich diet has a deep impact on a metabolically relevant tissue such as skeletal muscle. In this tissue, the consequences of high fructose feeding are altered glucose tolerance, elevated mitochondrial biogenesis and increased mitochondrial coupling. This latter modification could have a detrimental metabolic effect by causing oxidative stress and energy sparing that contribute to the high metabolic efficiency of fructose-fed rats.
\end{abstract}

Key words: Obesity: Insulin resistance: Ceramide

There is considerable evidence suggesting that in human subjects, elevated intake of sugar-sweetened beverages or added sugars in the form of sucrose and/or fructose is associated with increased body weight, the presence of unfavourable lipid levels, insulin resistance, fatty liver, type 2 diabetes, CVD and the metabolic syndrome ${ }^{(1)}$. Concerning the specific effects of the monosaccharide fructose on components of the metabolic syndrome, authors of recent reviews have concluded that high fructose intakes have substantial, even profound, deleterious metabolic consequences that possibly predispose individuals to chronic conditions such as type 2 diabetes and $\operatorname{CVD}^{(2,3)}$.

It has been previously shown that adult sedentary rats fed a high-fructose diet for 8 weeks exhibit hepatic insulin resistance, increased lipogenesis and lipid deposition ${ }^{(4)}$, in agreement with previous findings ${ }^{(5-8)}$ and with the fact that about $90 \%$ of fructose coming from the diet is metabolised in the liver $^{(2)}$. Increased hepatic lipid synthesis implies higher lipid circulation that could influence other tissues, such as skeletal muscle, and have a deep impact on whole-body metabolic homeostasis. Several data in the literature have underlined a potential link between insulin resistance and mitochondrial derangement in the skeletal muscle of human and animal models ${ }^{(9-11)}$, although this notion is challenged by several findings showing no mitochondrial defect with insulin resistance $^{(12-17)}$. On the basis of these considerations, the effect of long-term fructose feeding on skeletal muscle mitochondrial energetics was investigated. To this end, mitochondrial mass, oxidative capacity and efficiency in skeletal muscle were assessed. Measurements in isolated tissue were coupled with the determination of whole-body RMR and glucose tolerance.

\section{Research methods and procedures}

Male Sprague-Dawley rats (Charles River) of $90 \mathrm{~d}$ of age were caged singly in a temperature-controlled room $\left(23 \pm 1^{\circ} \mathrm{C}\right)$ with a $12 \mathrm{~h}$ light $-12 \mathrm{~h}$ dark cycle (06.30-18.30 hours) and divided into two groups with the same initial body weight that were fed a high-fructose or control diet (Mucedola 4RF21; Settimo Milanese) for 8 weeks. The composition of the two diets is given in Tables 1 and 2. Treatment, housing

Abbreviations: BSA, bovine serum albumin; FCCP, trifluorocarbonylcyanide phenylhydrazone; p-Akt, phosphorylated Akt; SOD, superoxide dismutase. 
Table 1. Composition of the experimental diets

\begin{tabular}{|c|c|c|}
\hline & Control diet & Fructose diet \\
\hline \multicolumn{3}{|l|}{ Components $(\mathrm{g} / 100 \mathrm{~g})$} \\
\hline Standard chow & $100 \cdot 0$ & $50 \cdot 5$ \\
\hline Sunflower oil & & 1.5 \\
\hline Casein & & $9 \cdot 2$ \\
\hline Alphacel & & $9 \cdot 8$ \\
\hline Fructose & & $20 \cdot 4$ \\
\hline Water & & $6 \cdot 4$ \\
\hline AIN-76 mineral mix ${ }^{*}$ & & 1.6 \\
\hline AIN-76 vitamin mix $\dagger$ & & 0.4 \\
\hline Choline & & $0 \cdot 1$ \\
\hline Met & & $0 \cdot 1$ \\
\hline Gross energy density $(\mathrm{kJ} / \mathrm{g})$ & $17 \cdot 2$ & $17 \cdot 2$ \\
\hline Metabolisable energy density $(\mathrm{kJ} / \mathrm{g}) \ddagger$ & $11 \cdot 1$ & $11 \cdot 1$ \\
\hline Protein $(\mathrm{J} / 100 \mathrm{~J})$ & $29 \cdot 0$ & $29 \cdot 0$ \\
\hline Lipids (J/100 J) & $10 \cdot 6$ & $10 \cdot 6$ \\
\hline Carbohydrates (J/100 J) & $60 \cdot 4$ & $60 \cdot 4$ \\
\hline Fructose & - & $30 \cdot 0$ \\
\hline Starch & $45 \cdot 3$ & $22 \cdot 8$ \\
\hline Sugars & $15 \cdot 1$ & $7 \cdot 6$ \\
\hline
\end{tabular}

AIN, American Institute of Nutrition.

${ }^{*}$ AIN, 1977.

†AIN, 1980.

‡Estimated by computation using values $(\mathrm{kJ} / \mathrm{g})$ for energy content as follows: protein 16.736 , lipid 37.656 and carbohydrate 16.736 .

and killing of animals met the guidelines set by the Italian Health Ministry. All experimental procedures involving animals were approved by 'Comitato etico-scientifico per la sperimentazione animale' of the University 'Federico II' of Naples.

At the end of the experimental period, the glucose tolerance test was carried out. Then, the animals were killed by decapitation, the skeletal muscle was harvested and the carcasses were used for body composition determination.

\section{$R M R$}

At the beginning and after 2, 46 and 8 weeks of the dietary treatment, $\mathrm{VO}_{2}$ of the rats was recorded with a monitoring system (Panlab s.r.l.) composed of a four-chamber indirect open-circuit calorimeter, designed for the continuous monitoring of up to four rats simultaneously. Measurements were performed every $15 \mathrm{~min}$ for $3 \mathrm{~min}$ in each cage. Food was withdrawn at 07.00 hours and mean values of a $2 \mathrm{~h}$ period (13.00-15.00 hours) were taken as an indicative of post-absorptive RMR.

\section{Glucose tolerance test and plasma NEFA}

Rats were fasted for $6 \mathrm{~h}$ from 08.00 hours. A point 0 sample was obtained from venous blood from a small tail clip and then glucose ( $2 \mathrm{~g} / \mathrm{kg}$ body weight) was injected intraperitoneally. Blood samples were collected after 20, 40, 60, 90, 120 and $150 \mathrm{~min}$. The blood samples were centrifuged at $1400 \mathbf{g}_{\text {av }}$ for $8 \mathrm{~min}$ at $4^{\circ} \mathrm{C}$. Plasma was removed and stored at $-20^{\circ} \mathrm{C}$ until used for determination of substrates and hormones. Plasma glucose concentration was measured by the colorimetric enzymatic method (Pokler Italia). Plasma insulin concentration was measured using an ELISA kit (Mercodia AB) in a single assay to remove inter-assay variations.
Plasma NEFA concentrations were measured by the colorimetric enzymatic method using a commercial kit (Randox Laboratories Limited).

\section{Body and skeletal muscle composition}

Guts were cleaned of undigested food and the carcasses were then autoclaved. After dilution (1:2 in distilled water) and subsequent homogenisation of the carcasses with a Polytron homogeniser (Kinematica), the resulting homogenates were frozen at $-20^{\circ} \mathrm{C}$ until the day of measurements. Duplicate samples of the homogenised carcasses were analysed for energy content by bomb calorimetry. To take into account the energy content of the harvested skeletal muscle, tissue samples were dried, the energy content was then measured with the bomb calorimeter and added to the body energy content. Total body and skeletal muscle lipid contents were measured by the Folch extraction method ${ }^{(18)}$. Total body protein content was determined using a formula relating total energy value of the carcass, energy derived from fat and energy derived from protein ${ }^{(19)}$; the energy values for body fat and protein were taken as 39.2 and $23.5 \mathrm{~kJ} / \mathrm{g}$, respectively ${ }^{(20)}$ Skeletal muscle TAG and cholesterol were measured by the colorimetric enzymatic method using commercial kits (SGM Italia), phospholipids were obtained by subtracting TAG and cholesterol contents from the total lipid content and glycogen was assessed by the direct enzymatic procedure ${ }^{(21)}$. Skeletal muscle ceramide content was evaluated by ELISA ${ }^{(22)}$ using ninety-six-well Polysorp plates (Nunc). In brief, skeletal muscle lipids $(70 \mu \mathrm{l}$ in methanol) were adsorbed to the well bottoms overnight at $4^{\circ} \mathrm{C}$. The plates were blocked with $10 \mathrm{~mm}-\mathrm{PBS}$, $140 \mathrm{~mm}-\mathrm{NaCl}$ and $0 \cdot 1 \%$ Tween ( $\mathrm{pH}$ 7.4) supplemented with $1 \%$ bovine serum albumin (BSA) for $1 \mathrm{~h}$ at $37^{\circ} \mathrm{C}$. The plates were then washed three times with $10 \mathrm{~mm}-\mathrm{PBS}, 140 \mathrm{~mm}-\mathrm{NaCl}$ and $0.05 \%$ Tween (pH 7.4) (Tween-PBS), and incubated with a monoclonal anti-ceramide antibody $(2 \mu \mathrm{g} / \mathrm{ml})$ for $1 \mathrm{~h}$ at $37^{\circ} \mathrm{C}$.

Table 2. Micronutrient composition of the experimental diets

\begin{tabular}{lcc}
\hline & Control diet & Fructose diet \\
\hline Thiamin $(\mathrm{mg} / 100 \mathrm{~g})$ & 1.72 & 1.11 \\
Riboflavin $(\mathrm{mg} / 100 \mathrm{~g})$ & 1.52 & 1.01 \\
Pyridoxine $(\mathrm{mg} / 100 \mathrm{~g})$ & 1.07 & 0.82 \\
Niacin $(\mathrm{mg} / 100 \mathrm{~g})$ & 2.5 & 2.5 \\
Calcium pantothenate $(\mathrm{mg} / 100 \mathrm{~g})$ & 2.5 & 1.9 \\
Folic acid $(\mathrm{mg} / 100 \mathrm{~g})$ & 0.23 & 0.20 \\
Biotin $(\mathrm{mg} / 100 \mathrm{~g})$ & 0.04 & 0.03 \\
Vitamin $\mathrm{B}_{12}(\mathrm{mg} / 100 \mathrm{~g})$ & 0.30 & 0.40 \\
Vitamin A palmitate $(\mathrm{mg} / 100 \mathrm{~g})$ & 0.27 & 0.43 \\
Vitamin $\mathrm{E}(\mathrm{mg} / 100 \mathrm{~g})$ & 5.2 & 6.4 \\
Vitamin $\mathrm{D}_{3}, \mathrm{cholecalciferol}(\mathrm{mg} / 100 \mathrm{~g})$ & 2.6 & 3.1 \\
Vitamin K $(\mathrm{mg} / 100 \mathrm{~g})$ & 0.32 & 0.22 \\
Calcium phosphate $(\mathrm{mg} / 100 \mathrm{~g})$ & 916.3 & 1262.7 \\
NaCl $(\mathrm{mg} / 100 \mathrm{~g})$ & 361.8 & 301.1 \\
$\mathrm{~K}(\mathrm{mg} / 100 \mathrm{~g})$ & 833.5 & 756.0 \\
Mg (mg/100 g) & 198 & 138 \\
Mn (mg/100 g) & 8.6 & 9.9 \\
Fe (mg/100 g) & 48 & 34 \\
Zn (mg/100 g) & 10.3 & 7.8 \\
Cu (g/100 g) & 2.57 & 1.78 \\
I (mg/100 g) & 0.1 & 0.07 \\
\hline
\end{tabular}


After three washings in Tween-PBS, peroxidase-conjugated goat anti-mouse $\operatorname{IgM}$ (1:5000 dilution) was incubated with the plates for $1 \mathrm{~h}$ at $37^{\circ} \mathrm{C}$. After four washings in Tween-PBS, the wells were incubated with $100 \mu$ l of a colour development solution $(20 \mathrm{mg} o$-phenylenediamine dihydrochloride in $50 \mathrm{ml}$

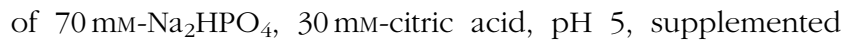
with $120 \mu \mathrm{l}$ of $3 \% \mathrm{H}_{2} \mathrm{O}_{2}$ ). After $15 \mathrm{~min}$ at $37^{\circ} \mathrm{C}$, the reaction was stopped by the addition of $50 \mu \mathrm{l}$ of $2.5 \mathrm{M}-\mathrm{H}_{2} \mathrm{SO}_{4}$ and absorbance was measured at $492 \mathrm{~nm}$. All tests were done in triplicate. Immunoreactivity was normalised to initial tissue weight. Negative control reactions included the omission of the primary antibody.

Metabolisable energy intake was determined as detailed previously $^{(23)}$, by subtracting the energy measured in the faeces and urine from the gross energy intake, determined from daily food consumption and gross energy density of the diet.

\section{Preparation of skeletal muscle isolated mitochondria} and measurements of mitochondrial oxidative capacities, degree of coupling and uncoupling effect of fatty acids

Hind leg muscles were rapidly removed and used for the preparation of isolated mitochondria. Hind leg muscles were freed of excess connective tissue, finely minced, washed in a medium containing $100 \mathrm{~mm}-\mathrm{KCl}, 50 \mathrm{~mm}-\mathrm{Tris}, \mathrm{pH}$ 7.5, $5 \mathrm{~mm}-\mathrm{MgCl}_{2}, 1 \mathrm{mm-EDTA}, 5 \mathrm{~mm}$-ethylene glycol tetraacetic acid and $0 \cdot 1 \%(\mathrm{w} / \mathrm{v})$ fatty acid-free BSA, and treated with protease nagarse $(1 \mathrm{mg} / \mathrm{g})$ for $4 \mathrm{~min}$. Tissue fragments were then homogenised with the above medium $(1: 8, \mathrm{w} / \mathrm{v})$ at $500 \mathrm{rpm}$ ( 4 strokes $/ \mathrm{min}$ ). Aliquots of the homogenate were withdrawn for measurements of state 3 mitochondrial respiration, while the rest was centrifuged at $3000 \boldsymbol{g}_{\text {av }}$ for $10 \mathrm{~min}$, the resulting supernatant was rapidly discarded and the pellet was resuspended and centrifuged at $500 \boldsymbol{g}_{\text {av }}$ for $10 \mathrm{~min}$. The supernatant was then centrifuged at $3000 \boldsymbol{g}_{\text {av }}$ for $10 \mathrm{~min}$, the pellet was washed once and resuspended in a suspension medium (250 mm-sucrose, 50 mm-Tris, $\mathrm{pH} 7.5$ and $0 \cdot 1 \%$ fatty acid-free BSA).

Oxygen consumption rate was measured polarographically with a Clark-type electrode (Yellow Springs Instruments) in a $3 \mathrm{ml}$ glass cell at $30^{\circ} \mathrm{C}$. Skeletal muscle homogenates or isolated mitochondria were incubated in a medium containing $30 \mathrm{~mm}-\mathrm{KCl}, 6 \mathrm{~mm}-\mathrm{MgCl}_{2}$,

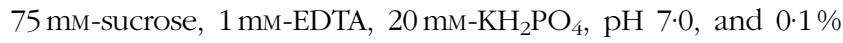
(w/v) fatty acid-free BSA, $\mathrm{pH} 7 \cdot 0$. The substrates used were $10 \mathrm{~mm}$-succinate $+3 \cdot 8 \mu \mathrm{m}$-rotenone, $10 \mathrm{~mm}$-glutamate $+2 \cdot 5 \mathrm{~mm}$ malate, $40 \mu \mathrm{m}$-palmitoyl-coenzyme $\mathrm{A}+2 \mathrm{~mm}$-carnitine $+2 \cdot 5$ mm-malate, $\quad 40 \mu \mathrm{m}$-palmitoyl-carnitine $+2.5 \mathrm{~mm}$-malate or 10 mm-pyruvate +2.5 mm-malate. State 3 oxygen consumption was measured in the presence of $0.3 \mathrm{~mm}-\mathrm{ADP}$.

The degree of coupling $(q)$ was determined in skeletal muscle mitochondria by applying equation 11 by Cairns et $a l^{(24)}$ :

$$
q=\sqrt{1-\left(J_{\mathrm{o}}\right)_{\mathrm{sh}} /\left(J_{\mathrm{o}}\right)_{\mathrm{unc}}}
$$

where $\left(J_{\mathrm{o}}\right)_{\mathrm{sh}}$ represents the oxygen consumption rate in the presence of oligomycin that inhibits ATP synthase and $\left(J_{\mathrm{O}}\right)_{\mathrm{unc}}$ is the uncoupled rate of oxygen consumption induced by trifluorocarbonylcyanide phenylhydrazone (FCCP), which dissipates the transmitochondrial proton gradient. $\left(J_{\mathrm{O}}\right)_{\mathrm{sh}}$ and $\left(J_{\mathrm{o}}\right)_{\text {unc }}$ were measured as above using succinate $10 \mathrm{~mm}+$ rotenone $3.75 \mu \mathrm{M}$ in the presence of oligomycin $(2 \mu \mathrm{g} / \mathrm{ml})$ or FCCP $(1 \mu \mathrm{M})$, respectively, both in the absence and presence of palmitate at a concentration of $45 \mu \mathrm{M}$.

The uncoupling effect of the fatty acid palmitate was assessed by measuring the mitochondrial membrane potential before and after the addition of increasing concentrations of palmitate. Mitochondrial membrane potential recordings were performed with safranin $\mathrm{O}$ using a JASCO dual-wavelength spectrophotometer $(511-533 \mathrm{~nm})$. Measurements were made at $30^{\circ} \mathrm{C}$ in a medium containing $30 \mathrm{~mm}-\mathrm{LiCl}, 6 \mathrm{~mm}-\mathrm{MgCl}_{2}$, 75 mm-sucrose, 1 mm-EDTA, 20 mm-Tris- $\mathrm{PO}_{4}$, $\mathrm{pH} 7 \cdot 0$, and $0 \cdot 1 \%$ $(\mathrm{w} / \mathrm{v})$ fatty acid-free BSA, $\mathrm{pH} 7 \cdot 0$, in the presence of succinate $(10 \mathrm{~mm})$, rotenone $(3.75 \mu \mathrm{m})$, oligomycin $(2 \mu \mathrm{g} / \mathrm{ml})$ and safranin $\mathrm{O}(83.3 \mathrm{nmol} / \mathrm{mg})$, both in the absence and after the addition of 15,30 and $45 \mu \mathrm{m}$-palmitate. Absorbance readings were transformed into $\mathrm{mV}$ membrane potential using the Nernst equation:

$$
\Delta \Psi=61 \mathrm{mV} \cdot \log \left(\left[\mathrm{K}^{+}\right]_{\text {in }} /\left[\mathrm{K}^{+}\right]_{\text {out }}\right) .
$$

Calibration curves made for each preparation were obtained from traces in which the extramitochondrial $\mathrm{K}^{+}$level $\left(\left[\mathrm{K}^{+}\right]_{\text {out }}\right)$ was altered in the $0 \cdot 1-20 \mathrm{~mm}$ range. The change in absorbance caused by the addition of $3 \mu \mathrm{M}$-valinomycin was plotted against $\left[\mathrm{K}^{+}\right]_{\text {out }}$. Then, $\left[\mathrm{K}^{+}\right]_{\text {in }}$ was estimated by extrapolation of the line to the zero uptake point.

\section{Western blot quantification of skeletal muscle Akt, phosphorylated Akt and mitochondrial cytochrome c}

Western blot analyses were carried out as described previously $^{(25)}$. Briefly, skeletal muscle samples were denatured in a buffer (60.0 mM-Tris, pH 6.8, $10 \%$ sucrose, $2 \%$ SDS and $4 \%$ $\beta$-mercaptoethanol) and loaded onto a $12 \%$ SDS-polyacrylamide gel. After the run in electrode buffer ( $50 \mathrm{~mm}$-Tris, $\mathrm{pH}$ $8 \cdot 3,384 \mathrm{~mm}$-glycine and $0 \cdot 1 \% \mathrm{SDS}$ ), the gels were transferred onto polyvinylidene difluoride membranes (Immobilon-P; Millipore) at $0.8 \mathrm{~mA} / \mathrm{cm}^{2}$ for $90 \mathrm{~min}$. The membranes were pre-blocked in blocking buffer (PBS, 5\% milk powder and $0.5 \%$ Tween 20) for $1 \mathrm{~h}$, and then incubated overnight at $4{ }^{\circ} \mathrm{C}$ with a polyclonal antibody for Akt and phosphorylated (p)-Akt (diluted 1:1000 in blocking buffer; Cell Signaling) or with a mouse monoclonal antibody for cytochrome $c$ (diluted 1:100 in blocking buffer; Biomol International). The membranes were washed three times for $12 \mathrm{~min}$ in $\mathrm{PBS} / 0.5 \%$ Tween-20 and three times for $12 \mathrm{~min}$ in PBS, and then incubated for $1 \mathrm{~h}$ at room temperature with an anti-mouse, alkaline phosphatase-conjugated secondary antibody (Promega). The membranes were washed as described above, rinsed in distilled water and incubated at room temperature with a chemiluminescent substrate (CDP-Star; Sigma-Aldrich). Data detection was carried out by exposing autoradiography films (Kodak; Eastman Kodak Company) to the membranes. Quantification 
of signals was carried out using Un-Scan-It gel software (Silk Scientific).

\section{Mitochondrial lipid peroxidation and superoxide dismutase specific activity}

Lipid peroxidation was determined according to Fernandes et al. ${ }^{(26)}$, by measuring thiobarbituric acid-reactive substances. Aliquots of mitochondrial suspensions were added to $0.5 \mathrm{ml}$ of ice-cold $40 \%$ TCA. Then, $2 \mathrm{ml}$ of $0.67 \%$ of aqueous thiobarbituric acid containing $0.01 \%$ of 2,6-di-tert-butyl-p-cresol were added. The mixtures were heated at $90^{\circ} \mathrm{C}$ for $15 \mathrm{~min}$, then cooled in ice for $10 \mathrm{~min}$ and centrifuged at $850 \mathrm{~g}$ for $10 \mathrm{~min}$. The supernatant fractions were collected and lipid peroxidation was estimated spectrophotometrically at $530 \mathrm{~nm}$. The amount of thiobarbituric acid-reactive substances formed was calculated using a molar extinction coefficient of $1.56 \times 10^{5}$ per $\mathrm{M} / \mathrm{cm}$ and expressed as nmol thiobarbituric acid-reactive substances/mg protein. Superoxide dismutase (SOD) specific activity was measured according to Flohè \& Otting ${ }^{(27)}$. Determinations were carried out spectrophotometrically $(550 \mathrm{~nm})$ at $25^{\circ} \mathrm{C}$ in a medium containing $0.1 \mathrm{~mm}$-EDTA, $2 \mathrm{~mm}-\mathrm{KCN}, \quad 50 \mathrm{~mm}-\mathrm{KH}_{2} \mathrm{PO}_{4}, \quad \mathrm{pH} \quad 7 \cdot 8,20 \mathrm{~mm}$-cytochrome $c$, $0 \cdot 1 \mathrm{~mm}$-xanthine and 0.01 units of xanthine oxidase, by monitoring the decrease in the reduction rate of cytochrome $c$ by superoxide radicals, generated by the xanthine-xanthine oxidase system. One unit of SOD activity is defined as the concentration of the enzyme that inhibits cytochrome $c$ reduction by $50 \%$ in the presence of xanthine + xanthine oxidase ${ }^{(27)}$

\section{Chemicals}

All chemicals utilised were of analytical grade and were purchased from Sigma.

\section{Statistical analysis}

Data are provided as means with their standard errors. Statistical analyses were performed using a two-tailed, unpaired, Student's $t$ test or repeated-measures two-way ANOVA for main effects and interactions followed by Bonferroni's posttest. All analyses were performed using GraphPad Prism 4 (GraphPad Software, Inc.).

\section{Results}

Body composition measurements carried out at the end of 8 weeks of the dietary treatment show that body energy was significantly higher in fructose-fed rats than in control rats (Table 3), due to an increase in energy from lipids but not in energy from proteins. Cumulative metabolisable energy intake over the 8 weeks of the dietary treatment was similar between the two groups (control rats 19600 (sEm 944) kJ; fructose-fed rats $19400(\operatorname{sEM} 453) \mathrm{kJ})$, thus indicating an increased metabolic efficiency in fructose-fed rats. As for the skeletal muscle composition, a significant increase in total lipids, TAG and ceramide was found in fructose-fed rats compared with the controls, while no variation was found in cholesterol,
Table 3. Energetic and metabolic characterisations in rats fed a high-fructose or control diet for 8 weeks

(Mean values with their standard errors, $n 6$ rats per group)

\begin{tabular}{lccccc}
\hline & \multicolumn{2}{c}{ Control } & & \multicolumn{2}{c}{ Fructose } \\
\cline { 2 - 3 } & Mean & SEM & & Mean & SEM \\
\hline Whole-body composition & & & & \\
$\quad$ Final weight (g) & 537 & 31 & & 538 & 32 \\
Energy (kJ) & 4690 & 252 & & $5227^{*}$ & 288 \\
Lipids (kJ) & 2521 & 130 & & $3042^{*}$ & 150 \\
Proteins (kJ) & 2155 & 112 & & 2209 & 107 \\
Skeletal muscle composition & & & & & \\
Lipids (mg/g) & 34.8 & 0.9 & & $41.2^{*}$ & 1.9 \\
TAG (mg/g) & 3.4 & 0.5 & & $7.0^{*}$ & 0.7 \\
Cholesterol (mg/g) & 1.5 & 0.1 & & 1.4 & 0.1 \\
Phospholipids (mg/g) & 31 & 2 & & 33 & 3 \\
Glycogen (mg/g) & 1.37 & 0.05 & & 1.47 & 0.03 \\
Ceramide (AU/g) & 0.018 & 0.001 & & $0.022^{*}$ & 0.001 \\
Plasma NEFA (mM) & 0.41 & 0.02 & & $0.53^{*}$ & 0.02 \\
\hline
\end{tabular}

$\mathrm{AU}$, absorbance units.

* Mean values were significantly different compared with the controls $(P<0.05$ two-tailed, unpaired Student's $t$ test).

phospholipids and glycogen. Rats fed a fructose-rich diet exhibited a significant increase in plasma NEFA (Table 3).

The results of the glucose tolerance test carried out in fructose-fed and control rats at the end of the diet treatment are presented in Fig. 1. The insulin response was significantly higher in fructose-fed rats compared with the controls (Fig. 1(a)), while no significant variation in plasma glucose levels was found, although the values for fructose-fed rats tended to be higher than those for control rats (Fig. 1(b)). Western blot analysis of the p-Akt:Akt ratio in skeletal muscle from fructose-fed and control rats showed no significant variation due to the dietary treatment (Fig. 1(c)). When p-Akt levels were normalised to insulin plasma levels, significantly lower values were found in fructose-fed rats compared with the controls (Fig. 1(c)). Finally, significantly lower RMR values were found in fructose-fed rats compared with the controls, starting from week 4 of the dietary treatment (Fig. 1(d)).

Mitochondrial oxidative capacities were assessed in isolated mitochondria from skeletal muscle using NAD, FAD and lipid substrates (Table 4) and the results obtained showed no significant variation in fructose-fed rats compared with the controls. Mitochondrial protein mass was assessed by measuring homogenate cytochrome $c$ content. The results reported in Table 4 showed that the cytochrome $c$ :actin ratio was significantly higher in fructose-fed rats compared with the controls. As a consequence, state 3 mitochondrial respiratory rates assessed in whole-tissue homogenates were found to be significantly higher in fructose-fed rats compared with the controls (Table 4).

Lipid peroxidation and SOD specific activity were measured and taken as an index of cellular oxidative damage and antioxidant defences, respectively (Table 4). A significant increase in lipid peroxidation and a significant decrease in SOD activity were found in fructose-fed rats compared with the controls.

Mitochondrial energetic efficiency was assessed through the measurement of state 4 respiration in the presence of 
(a)

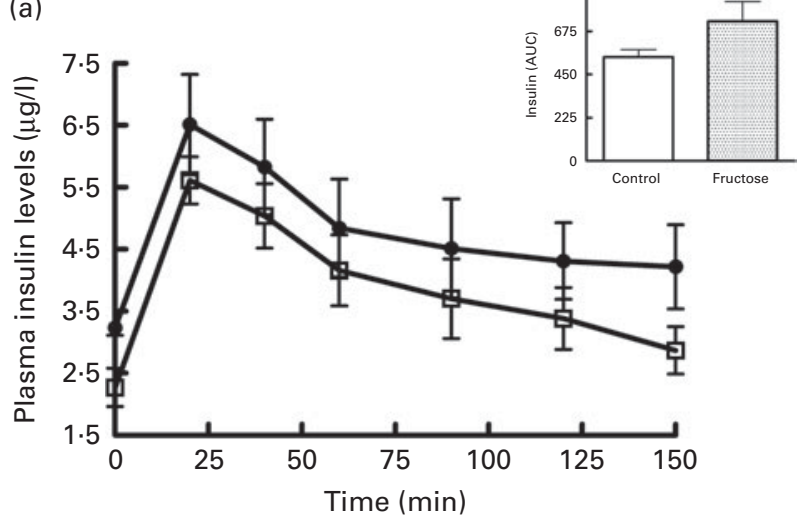

(c)

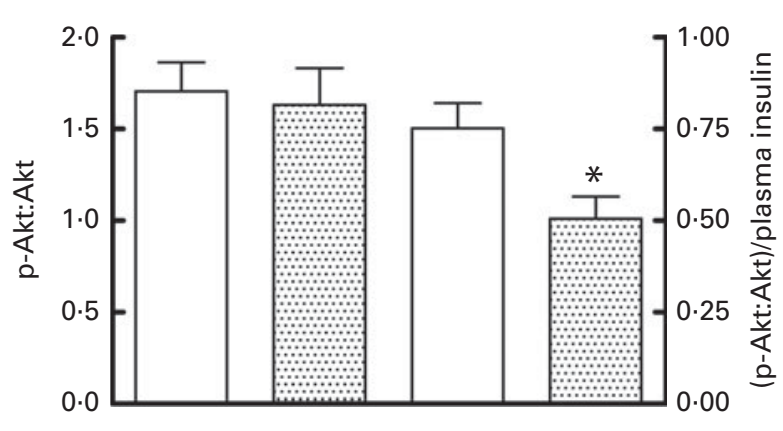

(b)

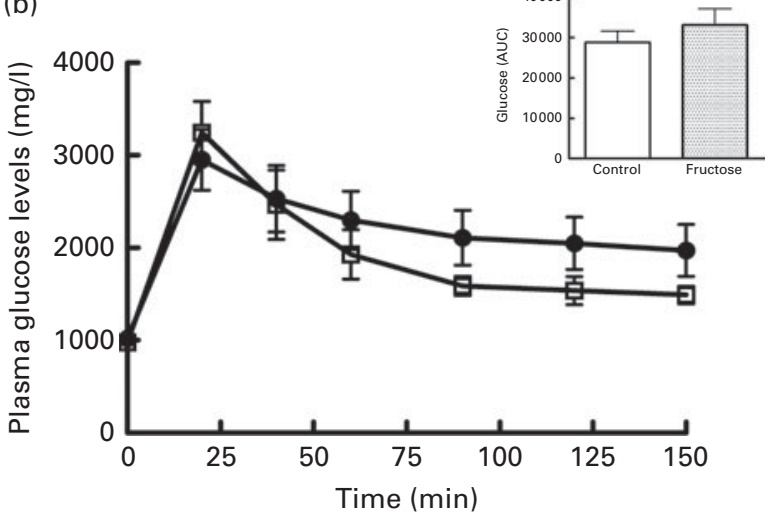

(d)

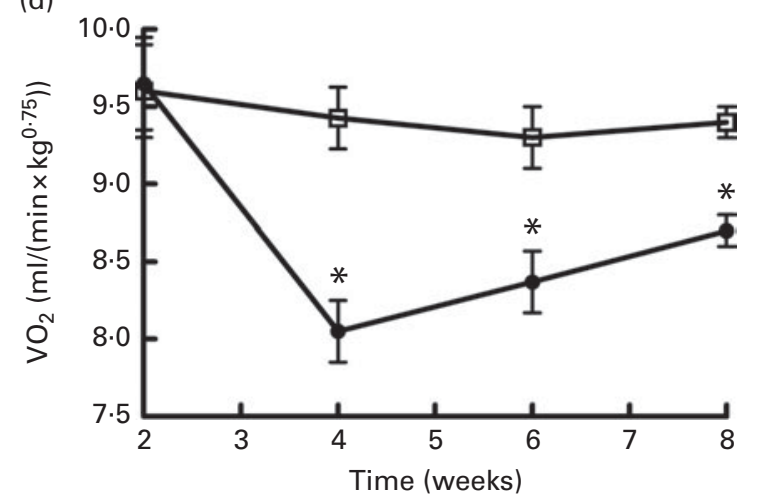

Fig. 1. Plasma (a) insulin and (b) glucose levels after a glucose load ( $\square$, control; $\rightarrow-$, fructose), (c) Western blot quantification of phosphorylated (p)-Akt:Akt levels and p-Akt:plasma insulin levels in skeletal muscle and (d) time course of RMR in fructose-fed ( $:$ ) or control ( $\square$ ) rats. Values are means ( 6 rats per group), with their standard errors represented by vertical bars. AUC was calculated using the trapezoid method. * Mean values were significantly different compared with the controls $(P<0.05$; two-tailed, unpaired Student's $t$ test for insulin AUC and p-Akt, repeated-measures two-way ANOVA for main effects and interactions followed by Bonferroni's post-test for RMR).

oligomycin and uncoupled respiration in the presence of FCCP, as well as through the evaluation of mitochondrial membrane potential in state 4 conditions, both in the absence and presence of physiological concentrations of palmitate. Oligomycin-induced state 4 respiration was significantly lower, both in the absence and presence of palmitate (Fig. 2(a)), while maximal FCCP-stimulated respiration was not affected by high fructose feeding, both in the absence and presence of palmitate (Fig. 2(b)), so that mitochondrial efficiency, assessed as the degree of coupling $(q)$, was significantly higher in fructose-fed rats compared with the controls, both in the absence and presence of palmitate (Fig. 2(c)). Mitochondria from fructose-fed rats were less responsive to the uncoupling effect of fatty acids, since the mitochondrial membrane potential in state 4 conditions was found to be significantly higher in fructose-fed rats compared with the controls, both in the absence and presence of increasing concentrations of the fatty acid palmitate (Fig. 2(d)).

\section{Discussion}

The main result of the present study is that long-term high fructose intake in adult rats induces an increase in skeletal muscle mitochondrial efficiency that could contribute to the condition of energy sparing (reduced RMR) found here.
First, the present results underline an important role for diet composition in the induction of obesity and indicate that not only a high lipid content but also the presence of simple sugars can affect body energy composition. In fact, despite the similar metabolisable energy intake during the whole 8-week period, fructose-fed rats exhibited higher body energy and the increase in body energy was almost exclusively as lipids. Interestingly, a parallel increase in circulating NEFA and skeletal muscle TAG and ceramide contents was found in fructose-fed rats. It should be noted that the percentage increase in plasma NEFA and muscle ceramide is similar, in agreement with previous results in obese human subjects of a significant correlation between muscle ceramide and plasma NEFA levels ${ }^{(28)}$.

Rats fed the fructose-rich diet also displayed reduced whole-body glucose tolerance, with a higher plasma insulinaemic response to a glucose load, results that are similar to those previously obtained in rats fed a high-fat diet ${ }^{(29)}$. Since glucose buffering capacity after a glucose load is primarily determined by the skeletal muscle metabolic response to insulin, downstream target of the insulin signalling pathway in the skeletal muscle of fructose-fed rats was investigated, and no difference was found in p-Akt kinase levels, but when the p-Akt kinase levels were normalised to plasma insulin 
Table 4. Mitochondrial oxidative capacities, mass and oxidative status in skeletal muscle from rats fed a high-fructose or control diet for 8 weeks

(Mean values with their standard errors, $n 6$ rats per group)

\begin{tabular}{|c|c|c|c|c|}
\hline & \multicolumn{2}{|c|}{ Control } & \multicolumn{2}{|c|}{ Fructose } \\
\hline & Mean & SEM & Mean & SEM \\
\hline \multicolumn{5}{|l|}{ State 3 respiration in isolated mitochondria ( $\mathrm{ng}$ atoms $\mathrm{O} /(\mathrm{min} \times \mathrm{mg}$ protein)) } \\
\hline Glutamate + malate & 846 & 55 & 861 & 55 \\
\hline Succinate + rotenone & 815 & 66 & 888 & 97 \\
\hline Palmitoyl-CoA + carnitine + malate & 341 & 28 & 365 & 23 \\
\hline Palmitoyl-carnitine + malate & 304 & 15 & 348 & 22 \\
\hline Pyruvate + malate & 788 & 41 & 819 & 60 \\
\hline \multicolumn{5}{|l|}{ State 3 respiration in whole-tissue homogenate ( $\mathrm{ng}$ atoms $\mathrm{O} /(\mathrm{min} \times \mathrm{g}$ wet tissue)) } \\
\hline Glutamate + malate & 3963 & 333 & $5456^{\star}$ & 451 \\
\hline Succinate + rotenone & 4172 & 337 & $5305^{\star}$ & 217 \\
\hline Palmitoyl-CoA + carnitine + malate & 1971 & 141 & $2474^{*}$ & 136 \\
\hline Palmitoyl-carnitine + malate & 1521 & 120 & $2013^{*}$ & 169 \\
\hline Pyruvate + malate & 4429 & 276 & $5723^{*}$ & 339 \\
\hline \multicolumn{5}{|l|}{ Mitochondrial mass } \\
\hline Cytochrome $c$ :actin ratio & $2 \cdot 78$ & 0.15 & $3 \cdot 40^{*}$ & 0.15 \\
\hline \multicolumn{5}{|l|}{ Mitochondrial oxidative status } \\
\hline Lipid peroxidation (nmol thiobarbituric acid-reactive substances/mg protein) & 4.99 & 0.25 & $5 \cdot 93^{*}$ & 0.30 \\
\hline Superoxide dismutase activity (U/mg protein) & $85 \cdot 1$ & $6 \cdot 0$ & $59 \cdot 2^{*}$ & $6 \cdot 0$ \\
\hline
\end{tabular}

* Mean values were significantly different compared with the controls $(P<0.05$; two-tailed, unpaired Student's $t$ test).

concentrations, significantly lower values were found in fructose-fed rats compared with the controls. This result is in agreement with increased skeletal muscle ceramide content, since it is well known that ceramide has been identified as

(a)

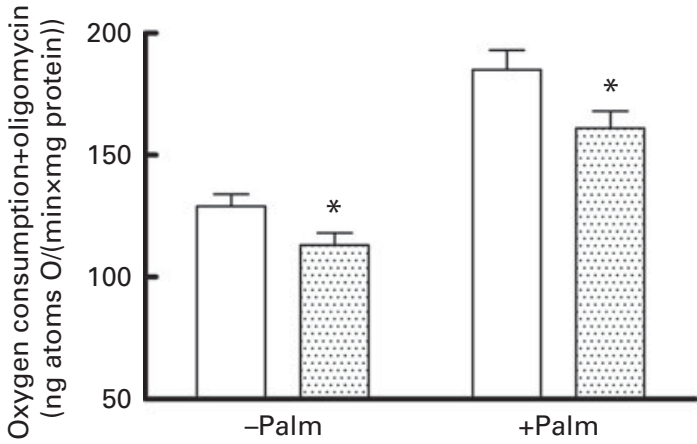

(c)

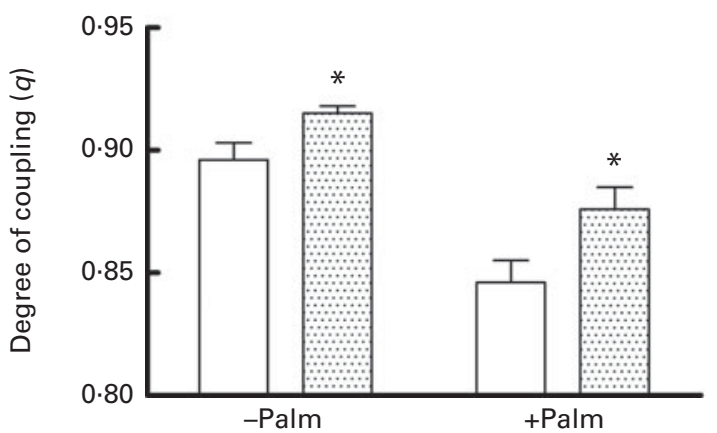

a key mediator of insulin resistance via inhibition of Akt phosphorylation $^{(30)}$, and indicates that insulin action is blunted in skeletal muscle from fructose-fed rats. However, this impairment is compensated by higher plasma insulin, so that the

(b)

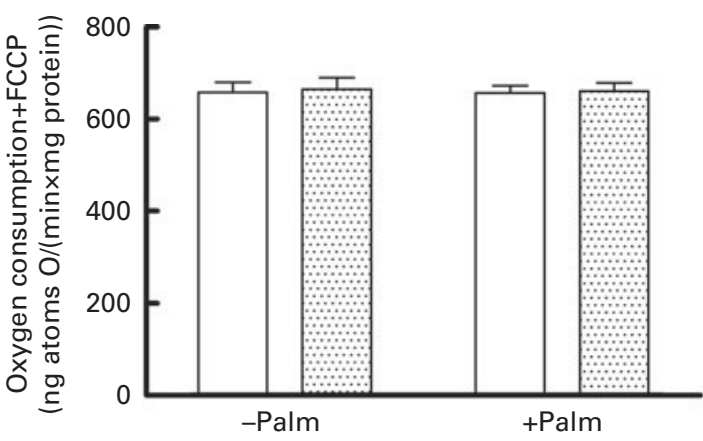

(d)

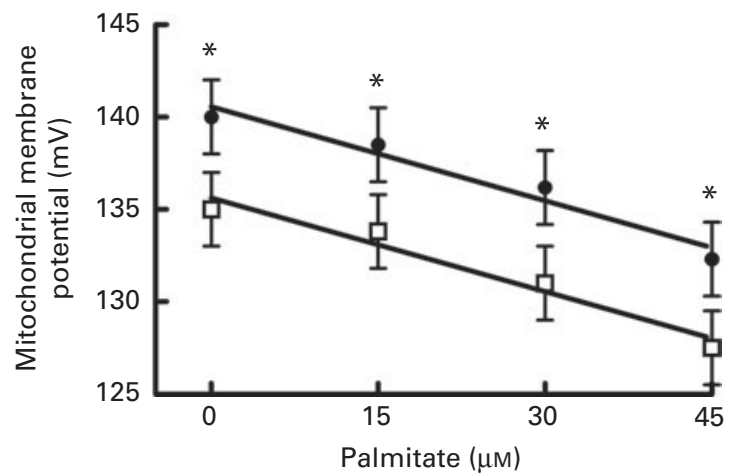

Fig. 2. Oxygen consumption in the (a) presence of oligomycin ( $\square$, control; $⿴ 囗 \mathrm{~g}$, fructose) or (b) uncoupled by trifluorocarbonylcyanide phenylhydrazone ( $\square$, control; $\square$, fructose), (c) degree of coupling values calculated from oxygen consumption in the presence of oligomycin and uncoupled by FCCP ( $\square$, control; $⿴$, fructose) and (d) membrane potential in state 4 conditions in the absence and presence of palmitate in hepatic mitochondria from fructose-fed (๑) and control $(\square)$ rats. Values are means ( $n 6$ per group), with their standard errors represented by vertical bars. ${ }^{*}$ Mean values were significantly different compared with the controls $(P<0.05$; two-way ANOVA for main effects and interactions followed by Bonferroni's post-test). 
final response of skeletal muscle cells to insulin is maintained. In agreement with this finding, tissue glycogen content does not vary in fructose-fed rats compared with the controls, in line with the well-known positive regulatory role of insulin in glycogen synthesis in this tissue ${ }^{(31)}$.

Whole-body insulin resistance is usually associated with alterations in the metabolic activity of skeletal muscle, and derangement in mitochondrial performance in skeletal muscle has been correlated with the development of insulin resistance ${ }^{(32)}$. Other studies have indicated that insulin resistance can be dissociated from mitochondrial respiratory capacity. Thus, in rat studies where the animals were given a high-fat diet, insulin resistance develops along with an increase in mitochondrial content and an increase in the capacity to oxidise fat ${ }^{(33-36)}$. Mitochondrial content inside the cell and oxidative phosphorylation efficiency are the main determinants of mitochondrial performance. Oxidative phosphorylation efficiency depends on the degree of coupling between oxygen consumption and ATP synthesis, which is always lower than 1 and can vary according to the metabolic needs of the cell ${ }^{(11)}$. It is well known that fatty acids can act as natural uncouplers of oxidative phosphorylation ${ }^{(37)}$. Therefore, mitochondrial mass and the degree of coupling, as well as the uncoupling effect of the fatty acid palmitate were assessed. The present results show that in fructose-fed rats, skeletal muscle mitochondrial mass increases, in agreement with the increased mitochondrial protein synthesis rate found in rats fed a high-fructose diet ${ }^{(38)}$. This increase in mitochondrial mass could be a compensatory mechanism to the increased fatty acid supply due to higher plasma NEFA levels found here, since it has been shown that raising plasma NEFA levels induces increased mitochondrial biogenesis in skeletal muscle ${ }^{(39)}$. However, the compensatory increase in mitochondrial mass probably failed to buffer NEFA oversupply, due to the increased mitochondrial coupling found in rats fed a fructose-rich diet, since in this condition, less amount of fuels are oxidised to obtain the same amount of ATP. Decreased substrate burning by skeletal muscle is supported by the decrease in RMR found here in fructose-fed rats compared with the controls, given that skeletal muscle accounts for about $30 \%$ of whole-body energy expenditure ${ }^{(40)}$. The failure of the mitochondrial compartment to face with the increased supply of lipid substrates in fructose-fed rats is also in agreement with their significantly higher skeletal muscle TAG, which imply an increase in intramuscular adipose tissue deposition. Intramuscular adipose tissue enlargement is considered among the determinants of insulin resistance ${ }^{(41)}$, through diacylglycerol and ceramide accumulation inside the skeletal muscle cells ${ }^{(42)}$.

Another unwanted consequence of the increased degree of coupling is higher mitochondrial free radical production (reactive oxygen species). In fact, the production of reactive oxygen species by the mitochondrial respiratory chain is higher when the membrane potential increases ${ }^{(43,44)}$, and reactive oxygen species production is indicated as one of the potential causes leading to insulin resistance ${ }^{(45,46)}$. For these reasons, the oxidative status of skeletal muscle mitochondria was also assessed and, in fructose-fed rats, signs of oxidative damage were found, together with the decreased activity of SOD, one of the enzymatic components of the antioxidant defence system. These results are in agreement with the impairment in insulin action found in this tissue, since it has recently been shown that the overexpression of mitochondrial SOD in skeletal muscle from rats fed a high-fat diet ameliorated the reduction in muscle glucose uptake and that this effect was mediated through an altered redox state s(47) $^{(20}$

In conclusion, the present results give evidence that a fructose-rich diet has a deep impact not only on liver tissue, which is responsible for about $90 \%$ of fructose metabolism, but also on another metabolically relevant tissue, the skeletal muscle. In this tissue, the consequences of high fructose feeding are decreased insulin signalling, stimulated mitochondrial biogenesis and increased mitochondrial coupling. This latter modification could have a detrimental metabolic effect by causing energy sparing that contributes to the high metabolic efficiency of fructose-fed rats.

\section{Acknowledgements}

The present study was supported by a grant from the University 'Federico II' of Naples and by P.O.R. Campania FSE 2007-13, Project CREME. The authors thank Dr Emilia De Santis for the skilful management of the animal house. S. I. designed and supervised the study. S. I. and G. L. obtained funding and provided administrative, technical and material support. R. C., F. B., P. C., A. M. and L. C. performed the animal experiments. R. C. and S. I. contributed to the analysis of data and the interpretation of the results. R. C., S. I. and G. L. wrote the draft of the manuscript. All the authors critically reviewed the manuscript. The authors declare that there is no conflict of interest.

\section{References}

1. Stanhope KL (2012) Role of fructose-containing sugars in the epidemics of obesity and metabolic syndrome. Annu Rev Med 63, 19.1-19.15.

2. Tappy L \& Le KA (2010) Metabolic effects of fructose and the worldwide increase in obesity. Physiol Rev 90, 23-46.

3. Dekker MJ, Su Q, Baker C, et al. (2010) Fructose: a highly lipogenic nutrient implicated in insulin resistance, hepatic steatosis, and the metabolic syndrome. Am J Physiol Endocrinol Metab 299, E685-E694.

4. Crescenzo R, Bianco F, Falcone I, et al. (2013) Increased hepatic de novo lipogenesis and mitochondrial efficiency in a model of obesity induced by diets rich in fructose. Eur J Nutr 52, 537-545.

5. Mayes PA (1993) Intermediary metabolism of fructose. Am J Clin Nutr 58, 754S-765S.

6. Herzberg GR \& Rogerson M (1988) Interaction of dietary carbohydrate and fat in the regulation of hepatic and extrahepatic lipogenesis in the rat. Br J Nutr 59, 233-241.

7. Carmona A \& Freedland RA (1989) Comparison among the lipogenic potential of various substrates in rat hepatocytes: the differential effects of fructose-containing diets on hepatic lipogenesis. J Nutr 119, 1304-1310.

8. Park J, Lemieux S, Lewis GF, et al. (1997) Chronic exogenous insulin and chronic carbohydrate supplementation increase 
de novo VLDL triglyceride fatty acid production in rats. J Lipid Res 38, 2529-2536.

9. Chaveza JA \& Summers SA (2010) Lipid oversupply, selective insulin resistance, and lipotoxicity: molecular mechanisms. Biochim Biophys Acta 1801, 252-265.

10. Patti ME \& Corvera S (2010) The role of mitochondria in the pathogenesis of type 2 diabetes. Endocr Rev 31, 364-395.

11. Johannsen DL \& Ravussin E (2009) The role of mitochondria in health and disease. Curr Opin Pharmacol 9, 780-786.

12. Boushel R, Gnaiger E, Schjerling P, et al. (2007) Patients with type 2 diabetes have normal mitochondrial function in skeletal muscle. Diabetologia 50, 790-796.

13. Rabol R, Hojberg PM, Almdal T, et al. (2009) Effect of hyperglycemia on mitochondrial respiration in type 2 diabetes. J Clin Endocrinol Metab 94, 1372-1378.

14. Asmann YW, Stump CS, Short KR, et al. (2006) Skeletal muscle mitochondrial functions, mitochondrial DNA copy numbers, and gene transcript profiles in type 2 diabetic and nondiabetic subjects at equal levels of low or high insulin and euglycemia. Diabetes 55, 3309-3319.

15. Larsen S, Ara I, Rabol R, et al. (2009) Are substrate use during exercise and mitochondrial respiratory capacity decreased in arm and leg muscle in type 2 diabetes. Diabetologia 52, $1400-1408$

16. Larsen S, Stride N, Hey-Mogensen M, et al. (2011) Increased mitochondrial substrate sensitivity in skeletal muscle of patients with type 2 diabetes. Diabetologia 54, 1427-1436.

17. Nair KS, Bigelow ML, Asmann YW, et al. (2008) Asian Indians have enhanced skeletal muscle mitochondrial capacity to produce ATP in association with severe insulin resistance. Diabetes 57, 1166-1175.

18. Folch J, Lees M \& Sloane Stanley GH (1957) A simple method for the isolation and purification of total lipides from animal tissues. J Biol Chem 226, 497-510.

19. Dulloo AG \& Girardier L (1992) Influence of dietary composition on energy expenditure during recovery of body weight in the rat: implications for catch-up growth and obesity relapse. Metabolism 41, 1336-1342.

20. Armsby HP (1917) The Nutrition of Farm Animals. New York: The Macmillan Company.

21. Roehrig KL \& Allred JB (1974) Direct enzymatic procedure for the determination of liver glycogen. Anal Biochem $\mathbf{5 8}$ 414-421.

22. Guilbault C, De Sanctis JB, Wojewodka G, et al. (2008) Fenretinide corrects newly found ceramide deficiency in cystic fibrosis. Am J Respir Cell Mol. Biol 38, 47-56.

23. Lionetti L, Mollica MP, Crescenzo R, et al. (2007) Skeletal muscle subsarcolemmal mitochondrial dysfunction in highfat fed rats exhibiting impaired glucose homeostasis. Int $J$ Obes 31, 1596-1604.

24. Cairns CB, Walther J, Harken AH, et al. (1998) Mitochondrial oxidative phosphorylation efficiencies reflect physiological organ roles. Am J Physiol 274, R1376-R1383.

25. Crescenzo R, Bianco F, Falcone I, et al. (2012) Mitochondrial energetics in liver and skeletal muscle after energy restriction in young rats. BrJ Nutr 108, 655-665.

26. Fernandes MAS, Custodio JBA, Santos MS, et al. (2006) Tetrandrine concentrations not affecting oxidative phosphorylation protect rat liver mitochondria from oxidative stress. Mitochondrion 6, 176-185.

27. Flohè L \& Otting F (1984) Superoxide dismutase assay. Meth Enzymol 105, 93-104.

28. Adams JM, Pratipanawatr T, Berria R, et al. (2004) Ceramide content is increased in skeletal muscle from obese insulinresistant humans. Diabetes 53, 25-31.
29. Crescenzo R, Bianco F, Falcone I, et al. (2008) Alterations in hepatic mitochondrial compartment in a model of obesity and insulin resistance. Obesity 16, 958-964.

30. Coen PM \& Goodpaster BH (2012) Role of intramyocellular lipids in human health. Trends Endocrinol Metab 23 391-398

31. Yeaman SJ, Armstrong JL, Bonavaud SM, et al. (2001) Regulation of glycogen synthesis in human muscle cells. Biochem Soc Trans 29, 537-541.

32. Pagel-Langenickel I, Bao J, Pang L, et al. (2010) The role of mitochondria in the pathophysiology of skeletal muscle insulin resistance. Endocr Rev 31, 25-51.

33. Turner N, Bruce CR, Beale SM, et al. (2007) Excess lipid availability increases mitochondrial fatty acid oxidative capacity in muscle: evidence against a role for reduced fatty acid oxidation in lipid-induced insulin resistance in rodents. Diabetes 56, 2085-2092.

34. Hancock CR, Han DH, Chen M, et al. (2008) High-fat diets cause insulin resistance despite an increase in muscle mitochondria. Proc Natl Acad Sci U S A 105, 7815-7820.

35. Garcia-Roves P, Huss JM, Han DH, et al. (2007) Raising plasma fatty acid concentration induces increased biogenesis of mitochondria in skeletal muscle. Proc Natl Acad Sci US A 104, 10709-10713.

36. Han DH, Hancock CR, Jung SR, et al. (2011) Deficiency of the mitochondrial electron transport chain in muscle does not cause insulin resistance. PLoS One 6, e19739.

37. Rial E, Rodríguez-Sánchez L, Gallardo-Vara E, et al. (2010) Lipotoxicity, fatty acid uncoupling and mitochondrial carrier function. Biochim Biophys Acta 1797, 800-806.

38. Chanseaume E, Giraudet C, Gryson C, et al. (2007) Enhanced muscle mixed and mitochondrial protein synthesis rates after a high-fat or high-sucrose diet. Obesity 15, 853-859.

39. Garcia-Roves P, Huss JM, Han DH, et al. (2007) Raising plasma fatty acid concentration induces increased biogenesis of mitochondria in skeletal muscle. Proc Natl Acad Sci US A 104, 10709-10713.

40. Rolfe DFS \& Brown GC (1997) Cellular energy utilization and molecular origin of standard metabolic rate in mammals Physiol Rev 77, 732-758.

41. Vettor R, Milan G, Franzin C, et al. (2009) The origin of intermuscular adipose tissue and its pathophysiological implications. Am J Physiol Endocrinol Metab 297, E987-E998.

42. Martins AR, Nachbar RT, Gorjao R, et al. (2012) Mechanisms underlying skeletal muscle insulin resistance induced by fatty acids: importance of the mitochondrial function Lipids Health Dis 11, 30-41.

43. Korshunov SS, Skulachev VP \& Starkov AA (1997) High protonic potential actuates a mechanism of production of reactive oxygen species in mitochondria. FEBS Lett $\mathbf{4 1 6}$, $15-18$.

44. Mailloux RJ \& Harper ME (2011) Uncoupling proteins and the control of mitochondrial reactive oxygen species production. Free Radic Biol Med 51, 1106-1115.

45. Houstis N, Rosen ED \& Lander ES (2006) Reactive oxygen species have a casual role in multiple forms of insulin resistance. Nature 440, 944-948.

46. Rains JL \& Jain SK (2011) Oxidative stress, insulin signaling, and diabetes. Free Radic Biol Med 50, 567-575.

47. Boden MJ, Brandon AE, Tid-Ang JD, et al. (2012) Overexpression of manganese superoxide dismutase ameliorates high-fat diet-induced insulin resistance in rat skeletal muscle. Am J Physiol Endocrinol Metab 303, E798-E805. 